\title{
5 Research Square

\section{Prognostic value of a four-level Chinese emergency triage for an Emergency Department: A prospective cohort study}

\section{Wenlong Huang}

Shenzhen Longhua New District People's Hospital https://orcid.org/0000-0003-1580-2914

\section{Xiaohua Xie}

Shenzhen Second People's Hospital

Haiyan Xiong

Shenzhen Longhua New District People's Hospital

Qiongling Liu

Guangdong Medical University

\section{Tan Wei}

Shenzhen Second People's Hospital

Yingchun Zeng ( $\nabla$ chloezengyc@hotmail.co.uk)

https://orcid.org/0000-0001-9250-4086

\section{Research article}

Keywords: Prognostic value, Admission rate, Four-level Chinese emergency triage, In-hospital mortality, Emergency department

Posted Date: September 27th, 2019

DOI: https://doi.org/10.21203/rs.2.15270/v1

License: (c) (i) This work is licensed under a Creative Commons Attribution 4.0 International License. Read Full License 


\section{Abstract}

Background In order to assign treatment priorities of patients who presenting to emergency departments (ED), various triage scales have been developed around the world. In china, four-tier triage scale was recommended by a national triage guideline that published by the central Chinese government. After that a four-tier triage system, used only in tertiary hospitals in Shenzhen, was published by the Public Hospital Administration of Shenzhen Municipalitfy in August, 2013 (Shenzhen Triage System, SZTS). But the ability in predicting admission and in-hospital mortality with the four-level triage scale for patients presented in emergency department was unclear. This research aimed to assess the prognostic value of the SZTS by examining the association between triage levels and clinical outcomes in adult patients.

Methods Patients 18 years of age or older, who were triaged category 1, 2 or 3 in the emergency department (ED) from May 17, 2017 until September 27, 2017, were enrolled. This study evaluated an association between triage level and in-hospital mortality, ICU admission and general ward admission by using multivariable logistic regression analysis. Receiver operating characteristic curves were used to assess the predictive ability of SZTS in determining in-hospital mortality, ICU admission and general ward admission.

Results There were 383 patients enrolled in the study. More urgent triage level were significantly related to a higher proportion of in-hospital mortality, ICU admission and general ward admission. The OR for inhospital mortality (OR 11.6, 95\% Cl 5.8-22.9, p<0.001), ICU admission (OR 14.4, 95\% Cl 7.4-27.7, p<0.001) and general ward admission (OR 10.6, 95\% $\mathrm{Cl} 1.4-79.2, \mathrm{p}=0.022)$ were greater at triage level 1 compared to triage level 2 and level 3. The AUC for in-hospital mortality, ICU admission and general ward admission prediction of SZTS was $0.838,0.830$ and 0.792 , respectively.

Conclusions This study suggests that triage levels were associated with general ward admission, ICU admission and in-hospital mortality, and SZTS showed good performance for clinical outcomes prediction.

\section{Background}

It is a global reality that emergency departments are overcrowded. In order to prioritize timely treatment for patients who present to emergency departments, triage scales have been implemented [1-5]. Hence, triage plays an important role in emergency medical care. Various triage scales have been developed around the world and are used to assign treatment priorities for patients presenting to emergency departments according to the severity of their condition [6-8]. In China, a national triage guideline was published by the central Chinese government in September, 2012 [9]. It promotes a four-tier triage scale that categorizes patients as endangered (level 1), critically ill (level 2), acute (level 3) and not acute (level 4). However, triage systems developed in different cities are not all the same in China $[10,11]$.

According to China's national standard, a triage standard for hospitals in Shenzhen was published by the Public Hospital Administration of Shenzhen Municipality in August, 2013 [12]. This standard also 
categorizes patients into four levels according to the presenting complaint and questions about potentially aggravating patient factors. Each triage level was assigned a color type, and each triage level indicates a maximum waiting time for the provision of emergency treatment. Red is assigned to triage level 1, which encompasses life-threatening cases and demands aggressive and immediate interventions. Red is also assigned to level 2, which includes serious cases with a potential threat to life requiring treatment within 10 minutes. Yellow is assigned to level 3 , which includes cases with urgent, acute symptoms without a potential threat to life requiring treatment within 30 minutes. Green is assigned to level 4, which includes patients with mild or non-urgent conditions without evidence of deterioration, requiring treatment within four hours.

Previous studies have shown that triage levels were related to certain patient outcome variables, such as hospital admission, ICU admission and in-hospital mortality [2,13-15]. Level 1, level 2 and level 3 are defined as urgent patients with a higher proportion of serious adverse events, such as hospital admission and mortality, compared with level 4 , which is defined as non-urgent patients $[13,16]$. However, no validation studies using data from real world practice have been conducted on the triage standard published by the Public Hospital Administration of Shenzhen Municipality. A study, using clinical data obtained in an ED, was therefore conducted to assess the validity of the triage standard published by the Public Hospital Administration of Shenzhen Municipality.

This study hypothesized there would be an association between triage levels and clinical outcomes. Since there is a lack of a gold standard for acuity, this study used markers such as general ward admission, ICU admission and in-hospital mortality, as was done in previous validation studies of triage systems. It was estimated that there would be a higher proportion of general ward admissions, ICU admissions and in-hospital mortality when the triage level was more urgent.

\section{Methods}

\section{Study design}

This study is a prospective observational study and was conducted in the ED of a tertiary hospital in Shenzhen, China. The aim of the study was to evaluate the relationship between the triage level and clinical outcomes of patients presenting to the Emergency Department. The protocol has been approved by the ethical committee of the Second People's Hospital of Shenzhen. The requirement for individual informed consent was waived due to the observational character of the study; and the data that we collected were originally intended for clinical practice.

\section{Study population}

The study was conducted in the First Affiliated Hospital of Shenzhen University. Including both adults and children, the hospital had 173,000 ED presentations in 2017 . Of this total, nearly 6,600 patients were admitted to the emergency treatment room. Patients presenting to the ED between 17 May 2017 and 27 
September 2017 were included. Eligible criteria for our study were as follows: (1) patients $\geq 18$ years of age (because SZTS is only suitable for adult patients); (2) patients were triaged category 1, 2 and 3. Exclusion criteria for this study were as follows: (1) Patients who had died prior to arrival in the ED. (2) Patients who needed ward admission, ICU admission or rescue according to a doctor's judgment, but ignored the doctor's advice and left the hospital for unspecified reasons. (3) Patients with insufficient information.

\section{Study procedure}

Patients who present to the ED must be triaged by the triage nurse, who has more than five years of experience in nursing in the ED, using SZTS. SZTS consists of seven measurable indicators (respiratory rate, heart rate, systolic blood pressure, diastolic blood pressure, temperature, percutaneous oxygen saturation and AVPU score), 34 main complaint groups and 179 complaints. This information was entered in the electronic database by triage nurses. Then patients were assigned to one of four levels of urgency by the SZTS, which recommends a maximum time for receiving care by physicians. Patients were sent to a different area according to their triage level. Level 1 (red): life threatening and requires immediate interventions; Level 2 (red): critically ill and requires treatment within 10 minutes; Level 3 (yellow): urgent and requires treatment within 30 minutes; Level 4 (green): non-urgent and requires treatment within four hours. However, the triage nurse can modify the triage level for certain groups of complaints. For a patient presenting to our hospital, all information, such as identifying information, triage level and clinical outcome, was registered in the hospital information system. Therefore, the baseline characteristics, such as demographics, in-hospital mortality and admission to the intensive care unit was obtained through the information system for this analysis.

\section{Outcomes}

This study used a composite of admission to the ICU and in-hospital mortality as the primary outcome. The secondary outcome was using SZTS to predict discharge from hospital or admission to the general ward unit.

\section{Statistical analysis}

First, baseline characteristics and clinical parameters of patients triaged to level 1, level 2 and level 3 were compared using the $t$ test for normal distribution data and Kruskal-Wallis rank sum test for non-normal distribution data. Second, the association between triage level and in-hospital mortality, ICU admission and general ward admission was examined using multivariable logistic regression analysis with adjustment for age and gender. This study also calculated the proportion of in-hospital mortality, admission to ICU and predicted admission to the general ward in each triage level. Finally, the predictive value of SZTS for in-hospital mortality, admission to ICU and admission to the general ward were 
examined using receiver operating characteristic (ROC) curves. $P<0.05$ was considered as statistically significant. EPidata3.1 was used for data entry, and then exported to tab-delimited text files. All analyses were performed using R (http://www.R-project.org) and EmpowerStats software (www.empowerstats.com, X\&Y Solutions, Inc., Boston, MA).

\section{Results}

A total of 516 patients met the eligibility criteria, with 133 patients excluded. Of the patients who were excluded from the study, 10 patients had already died when sent to the ED, while 46 patients refused treatment when in the ED and 65 patients refused treatment after admittance to the ward or ICU; 12 patients were also excluded due to insufficient information (Figure 1). Finally, 383 patients were included in this research. Baseline characteristics of patients triaged to level 1, level 2 and level 3 are presented (Table 1). Among the 383 enrolled patients, the number of patients triaged to level 1, level 2 and level 3 was 69, 208 and 106, respectively. A more urgent triage level was significantly related to older age, higher general ward admission, ICU admission and in-hospital mortality. Nervous system diseases, respiratory diseases, digestive diseases and cardiovascular diseases were the main diagnoses of patients presenting to the ED.

\section{Comparison of clinical parameters of patients triaged to level 1, level 2 and level 3}

According to the triage level, patients were divided into three groups. More urgent triage levels were significantly related to higher heart rate, respiratory rate, a longer length of stay, lower percutaneous oxygen saturation, systolic blood pressure and diastolic blood pressure. There were differences in patient mental health status in the three different groups. The proportion of patients triaged to Level 1 with a disorder of consciousness was $84.1 \%$, however, the proportion of patients triaged to Level 3 was $2.8 \%$. Detailed physiology parameters for the three groups are shown in Table 2.

\section{SZTS predictive ability}

The receiver operating characteristics curve (AUC) was used for SZTS clinical outcome prediction (Figure $2,3,4)$. The AUC for in-hospital mortality, ICU admission and general ward admission prediction of the SZTS was $0.838,0.830$ and 0.792 , respectively. Logistic regression analysis indicated that SZTS levels were significantly associated with patient in-hospital mortality, ICU admission and general ward admission (Table 2). Patients with more urgent triage levels had a higher risk of in-hospital mortality, ICU admission and general ward admission. Comparing triage level 1 with triage level 2, the OR for in-hospital mortality (OR 11.6, 95\% Cl 5.8-22.9, p<0.001), ICU admission (OR 14.4, 95\% Cl 7.4-27.7, p<0.001) and general ward admission (OR 10.6, 95\% $\mathrm{Cl} 1.4-79.2, \mathrm{p}=0.022)$ were greater. When adjusted for age and gender, OR for comparing triage level 1 with triage level 2 were greater as well. The more urgent the triage 
level, the higher the proportion of in-hospital mortality, ICU admission and general ward admission. The proportion of in-hospital mortality, ICU admission and general ward admission in patients triaged to level 1 was $98.6 \%, 76.8 \%$ and $50.7 \%$,respectively. When patients were triaged to level 2 and level 3 , the proportion of in-hospital mortality, ICU admission and general ward admission was as follows ( $86.5 \% \mathrm{vs}$ $43.4 \%, 18.7 \%$ vs $0.9 \%, 18.1 \%$ vs $0.0 \%)$.

\section{Discussion}

In this study, the results showed that triage level as defined in the triage system is closely related to clinical outcomes, such as in-hospital mortality and admission to the ICU. Similarly, other studies also show that the urgency categories are related to patient outcomes [8,17-21].

This study indicated that in-hospital mortality and admission decreased with triage urgency. Patients triaged to level 1 had the highest proportion of in-hospital mortality and admission. More than half of patients triaged to level 1 died during hospitalization and $76.8 \%$ were admitted to the ICU. Kwak et al [17] found an increased ratio of in-hospital mortality and ICU admission with increased urgency in the Emergency Severity Index (ESI). Patients triaged to ESI level 1 had the highest proportion of in-hospital mortality and admission. The proportion of composite outcome (in-hospital mortality and ICU admission) in patients triaged to ESI level 1, level 2, level 3, level 4 and level 5 were $16.9 \%$ (49.0\%), 3.4\% (13.9\%), $0.4 \%$ (1.8\%), $0.0 \%(0.1 \%), 0.0 \%(0.1 \%)$, respectively.

Another study conducted by van der Wulp et al [22] reported the associations between admission, mortality and urgency categories of two triage systems (ESI and the Manchester Triage System MTS). Percentage of mortality per triage category varied, with patients triaged in ESI category 1, ESI category 2, ESI category 3 , ESI category 4 and ESI category $5,82.1 \%, 10.7,7.1 \%, 0.0 \%, 0.0 \%$, respectively, and patients triaged in the MTS categories of Red, Orange, Yellow, Green and Blue were 75.9\%, 20.7, 3.4, $0.0 \%, 0.0 \%$, respectively. However, we found that the rates of mortality and admission in patients in the same triage category varied in the different research studies. First, these differences could vary according to study populations, for example depending on factors such as age, which was an important predictor of urgency. A study conducted by Kim et al [23] showed that patient age and disease type when presenting to the ED were significant predictors in predicting patient admission to hospital. In addition, triage systems generated from different countries with different populations and economic levels may also have contributed to the diverse results.

In this study, the triage system demonstrated good predictive ability for in-hospital mortality, ICU admission and general ward admission prediction ( $A U C=0.838,0.830,0.792$ respectively). Similar results were shown in the validation studies of other triage systems [2,24]. A retrospective study to assess the performance of the Manchester Triage System in older patients demonstrated that MTS predicted hospital admission and in-hospital mortality with AUC values of 0.74 and 0.71 , respectively [24]. Kuriyama et al [2] conducted a research that enrolled patients ages 16 years or older, and evaluated the association between Japan Acuity and Triage Scale triage levels and overall admission and ICU 
admission. The results showed the AUC values for overall admission and ICU admission prediction were 0.726 and 0.792 . Another study that evaluated the performance of the Emergency Severity Index in patients older than 15 years indicated good performance for composite outcome (in-hospital mortality and ICU admission) and in-hospital mortality prediction with AUC values of 0.786 and 0.778 [17].

However, in a prospective cohort study the Manchester Triage System showed fair performance for predicting ICU admission with AUC values of 0.68 , but not for predicting 30 -day mortality with AUC values of 0.55 [25]. All of the above studies have shown that the triage systems' predictive ability for patient clinical outcomes varied from fair to good, and the AUC values were higher in our study. The results indicate that the triage system used in our study had good performance for clinic outcome prediction.

This study showed that the higher the urgency triage categories of patients, the greater their risk of hospitalization and progression to death. Compared with patients triaged at level 2, patients classified as level 1 had 9.6 times more chance of general ward unit admission, 14.4 times more chance of ICU admission and 10.6 times more chance of in-hospital mortality. There are previously published studies of other triage systems indicating similar results $[17,18,22]$. A retrospective study conducted on patients aged 15 years and older showed that patients triaged to ESI level 1 had 26.47 times more chance of inhospital mortality and 40.61 times more chance of composite outcomes than patients triaged to ESI level 3 [17]. The triage system in our study was a four-level triage system, which was different from the fivelevel triage systems. Moreover, the references in those studies were also different, which made comparison through odds ratio (OR) difficult. However, the higher the urgency of triage level, the higher the OR for ICU admission and in-hospital mortality is the common conclusion.

There are some limitations in the study. Firstly, SZTS is a triage system that is only used in hospitals in Shenzhen, China. Therefore, our conclusions may not be generalizable to other settings. Secondly, it was conducted as a single-center, observational study and at a tertiary hospital. Patient triage level is closely related to the experience of the triage nurse, and may therefore have affected the performance of the SZTS for clinical outcome prediction. Third, in some cases triage level may fail to indicate the severity of patient illness. A patient with severe flank pain (VAS 10/10) was sent to the ED. He was assessed as triage level 2 and was sent to the emergency treatment room. However, after adequate pain control the patient was discharged home and was ultimately diagnosed with urolithiasis with no high-risk features. According to the SZTS, the patient should have been assessed into level 3 . Thus, considering the limitations of the research, multi-centric prospective cohort studies are warranted to verify the efficacy of the findings.

\section{Conclusions}

In summary, this study indicated a strong relationship between triage level and patient outcome, and the SZTS demonstrated good prognostic value for Emergency Department clinical outcomes prediction.

\section{Abbreviations}




\title{
AUC, Area under curve;
}

\section{ED, Emergency Department;}

\section{ESI, Emergency Severity Index;}

\author{
ICU, Intensive Care Unit;
}

ROC, receiver operating characteristic;

SZTS, Shenzhen Triage System

\section{Declarations}

\section{Ethics approval and consent to participate}

The study was approved by the ethical committee of the Second People's Hospital of Shenzhen (No. 20141201005). The requirement for individual informed consent was waived due to the observational character of the study; and the data that we collected were originally intended for clinical practice.

\section{Consent for publication}

All authors consent to the publication of this manuscript.

\section{Availability of data and material}

The data used in this study is available from the corresponding author on reasonable request.

\section{Competing interests}

The authors declare no conflict of interest.

\section{Funding}

This study was partly supported by the Science and Technology Department of Guangdong Province, China (No. 2014A020212574) for supporting data collection, and was also funded by Research Projects of Guangzhou Health Commission (No. 20191A011088) for supporting data analysis of this study. 


\section{Author Contributions}

Study concept and design: Xiaohua Xie, Wenlong Huang, Yingchun Zeng; acquisition of data: Wenlong Huang; analysis and interpretation of data: all authors; Project administration: Xiaohua Xie, Yingchun Zeng; first drafting of the manuscript: Wenlong Huang, Xiaohua Xie; revision of the important intellectual content in the manuscript: all authors; statistical analysis: Wenlong Huang.

\section{Acknowledgment}

We sincerely thank Xinglin Chen for her excellent assistance with the statistical analyses.

\section{References}

1. Hammad K, Peng L, Anikeeva O, Arbon P, Du H, Li Y. Emergency nurses' knowledge and experience with the triage process in Hunan Province, China. Int Emerg Nurs 2017;35:25-29.

2.Kuriyama A, Ikegami T, Kaihara T, Fukuoka T, Nakayama T. Validity of the Japan Acuity and Triage Scale in adults: a cohort study. Emerg Med J 2018;35(6):384-388.

3.Mirhaghi A, Heydari A, Mazlom R, Ebrahimi M. The Reliability of the Canadian Triage and Acuity Scale: Meta-analysis. N Am J Med Sci 2015;7(7):299-305.

4.Ebrahimi M, Heydari A, Mazlom R, Mirhaghi A. The reliability of the Australasian Triage Scale: a metaanalysis. World J Emerg Med 2015;6(2):94-99.

5.Mirhaghi A, Mazlom R, Heydari A, Ebrahimi M. The reliability of the Manchester Triage System (MTS): a meta-analysis. J Evid Based Med 2017;10(2):129-135.

6.Krey J. Triage in emergency departments. Comparative evaluation of 4 international triage systems. Med Klin Intensivmed Notfmed 2016;111(2):124-133.

7.Chang W, Liu HE, Goopy S, Chen LC, Chen HJ, Han CY. Using the Five-Level Taiwan Triage and Acuity Scale Computerized System: Factors in Decision Making by Emergency Department Triage Nurses. Clin Nurs Res 2017;26(5):651-666.

8.Zachariasse JM, van der Hagen V, Seiger N, Mackway-Jones K, van Veen M, Moll HA. Performance of triage systems in emergency care: a systematic review and meta-analysis. BMJ Open 2019;9(5):e026471.

9.China MoHotPsRo. The design of normative flow of emergency department. 2012.

10.Peng L, Hammad K. Current status of emergency department triage in mainland China: A narrative review of the literature. Nurs Health Sci 2015;17(2):148. 
11.Ng CJ, Chien CY, Seak JC, Tsai SL, Weng YM, Chaou CH, et al. Validation of the five-tier Taiwan Triage and Acuity Scale for prehospital use by Emergency Medical Technicians. Emerg Med J 2019;36(8):472478.

12.Muncicipality PHAoS. The hospital emergency triage guidelines (Trial). 2013.

13.Yuksen C, Sawatmongkornkul S, Suttabuth S, Sawanyawisuth K, Sittichanbuncha Y. Emergency severity index compared with 4-level triage at the emergency department of Ramathibodi University Hospital. Asian Biomedicine 2016;10(2):155-161.

14. Zachariasse JM, Seiger N, Rood PP, Alves CF, Freitas P, Smit FJ, et al. Validity of the Manchester Triage System in emergency care: A prospective observational study. PloS one 2017;12(2):e0170811.

15.Mullan PC, Torrey SB, Chandra A, et al. Reduced overtriage and undertriage with a new triage system in an urban accident and emergency department in Botswana: a cohort study. Emerg Med J 2014;31(5):356-360.

16.Xie X, Huang W, Liu Q, Tan W, Pan L, Wang L, et al. Prognostic value of Modified Early Warning Score generated in a Chinese emergency department: a prospective cohort study. BMJ Open 2018;8(12):e024120.

17.Kwak H, Suh GJ, Kim T, Kwon WY, Kim KS, Jung YS, et al. Prognostic performance of Emergency Severity Index (ESI) combined with qSOFA score. Am J Emerg Med 2018;36(10):S0735675718301025.

18.Becker JB, Lopes MC, Pinto MF, Campanharo CR, Barbosa DA, Batista RE. Triage at the Emergency Department: association between triage levels and patient outcome. Rev Esc Enferm USP 2015;49(5):783.

19. Gravel J, Fitzpatrick E, Gouin S, Millar K, Curtis S, Joubert G, et al. Performance of the Canadian Triage and Acuity Scale for children: a multicenter database study. Ann Emerg Med 2013;61(1):27-32.e23.

20.Yates MT, Ishikawa T, Schneeberg A, Brussoni M. Pediatric Canadian Triage and Acuity Scale (PaedsCTAS) as a Measure of Injury Severity. Int J Environ Res Public Health 2016;13(7):659.

21.Allon R, Feldman O, Karminsky A, Steinberg C, Leiba R, Shavit I. Validity of the Pediatric Canadian Triage Acuity Scale in a tertiary children's hospital in Israel. Eur J Emerg Med 2018;25(4):270-273.

22. van der Wulp I, Schrijvers AJ, van Stel HF. Predicting admission and mortality with the Emergency Severity Index and the Manchester Triage System: a retrospective observational study. Emerg Med J 2009;26(7):506-509.

23.Kim SW, Li JY, Hakendorf P, Teubner DJ, Ben-Tovim DI, Thompson CH. Predicting admission of patients by their presentation to the emergency department. Emerg Med Australasia 2014;26(4):361-367. 
24.Brouns SHA, Mignot-Evers L, Derkx F, Lambooij SL, Dieleman JP, Haak HR. Performance of the Manchester triage system in older emergency department patients: a retrospective cohort study. BMC Emerg Med 2019;19(1):3.

25.Steiner D, Renetseder F, Kutz A, Haubitz S, Faessler L, Anderson JB, et al. Performance of the Manchester Triage System in Adult Medical Emergency Patients: A Prospective Cohort Study. J Emerg Med 2016;50(4):678-689.

\section{Tables}

Table 1 Baseline characteristics of patients triaged to level 1, level 2 and level 3

\begin{tabular}{|c|c|c|c|c|}
\hline \multirow[t]{2}{*}{ Characteristics } & Level 1 & \multirow{2}{*}{$\begin{array}{c}\text { Level } 2 \\
\text { n■208 }\end{array}$} & \multirow{2}{*}{$\begin{array}{r}\text { Level } 3 \\
\text { n®106 }\end{array}$} & \multirow[t]{2}{*}{$P$ value } \\
\hline & nロ69 & & & \\
\hline Age & $64.45(19.4)$ & $60.3(17.3)$ & $55.0(18.7)$ & 0.003 \\
\hline Gender n ( $\square)$ & & & & 0.746 \\
\hline Male & $47(68.1)$ & $135(64.9)$ & $73(68.9)$ & \\
\hline Female & $22(31.9)$ & $73(35.1)$ & $33(31.1)$ & \\
\hline Ethnicity n (口) & & & & 0.125 \\
\hline Han & $68(98.6)$ & 206 (99) & $102(96.2)$ & \\
\hline Hui & $0(0.0)$ & $1(0.5)$ & $0(0.0)$ & \\
\hline Manchu & $1(1.4)$ & $1(0.5)$ & $4(3.8)$ & \\
\hline Means of arrival n ( () & & & & $<0.001$ \\
\hline Walking & $1(1.4)$ & 66 (31.7) & $56(52.8)$ & \\
\hline Wheelchair & $2(2.9)$ & $4(1.9)$ & $2(1.9)$ & \\
\hline Ambulance & 66 (95.7) & $138(66.3)$ & $48(45.3)$ & \\
\hline Triage n ( $(\mathrm{)})$ & & & & $<0.001$ \\
\hline Discharged from ED & $0(0.0)$ & $7(3.4)$ & $31(29.2)$ & \\
\hline Observation Room & $1(1.4)$ & $21(10.1)$ & $29(27.4)$ & \\
\hline Ward admission & $15(21.7)$ & $141(67.8)$ & $45(42.5)$ & \\
\hline ICU admission & $45(65.2)$ & 37 (17.8) & $1(0.9)$ & \\
\hline Died in ED & $8(11.6)$ & $2(1.0)$ & $0(0.0)$ & \\
\hline Disease type n ( $(\square)$ & & & & $<0.001$ \\
\hline Respiratory & $16(23.2)$ & $25(12.0)$ & $13(12.3)$ & \\
\hline Digestive & $2(2.9)$ & $13(6.2)$ & $21(19.8)$ & \\
\hline Cardiovascular & $5(7.2)$ & $60(28.8)$ & $17(16.0)$ & \\
\hline Nervous system & $20(29.0)$ & $60(28.8)$ & $19(17.9)$ & \\
\hline Hematological & $0(0.0)$ & $0(0.0)$ & $3(2.8)$ & \\
\hline Endocrinologic, metabolism & $2(2.9)$ & $0(0.0)$ & $1(0.9)$ & \\
\hline Urinary & $2(2.9)$ & $10(4.8)$ & $3(2.8)$ & \\
\hline Trauma & $7(10.1)$ & $16(7.7)$ & $5(4.7)$ & \\
\hline Others & $15(21.7)$ & $24(11.5)$ & $24(22.6)$ & \\
\hline
\end{tabular}

Abbreviations: ICU intensive care unit, ED emergency department. 
Table 2 Clinical parameter comparison of patients triaged to level 1, level 2 and level 3

\begin{tabular}{|c|c|c|c|c|}
\hline \multirow[t]{2}{*}{ Parameters } & Level 1 & Level 2 & Level 3 & \multirow[t]{2}{*}{$P$ value } \\
\hline & nロ69 & $\mathrm{n} \square 208$ & $\mathrm{n} \square 106$ & \\
\hline Age mean (SD) & $64.45(19.4)$ & $60.3(17.3)$ & $55.0(18.7)$ & 0.003 \\
\hline Gender $n(\square)$ & & & & 0.746 \\
\hline Male & $47(68.1)$ & $135(64.9)$ & $73(68.9)$ & \\
\hline Female & $22(31.9)$ & $73(35.1)$ & $33(31.1)$ & \\
\hline \multicolumn{5}{|l|}{ Physiology } \\
\hline $\mathrm{T}\left({ }^{\circ} \mathrm{C}\right)$ mean $(\mathrm{SD})$ & $37.4(1.3)$ & $37.2(0.9)$ & $37.0(0.8)$ & 0.404 \\
\hline SBP $(\mathrm{mmHg})$ & $126.1(39.7)$ & $137.8(33.3)$ & $140.8(28.6)$ & 0.036 \\
\hline \multicolumn{5}{|l|}{ Mean (SD) } \\
\hline DBP (mmHg) mean (SD) & $72.9(26.4)$ & 82.3(20.1) & $81.3(18.0)$ & 0.01 \\
\hline Heart rate (bpm) mean (SD) & $106.7(37.9)$ & $97.2(31.6)$ & $84.0(17.9)$ & $<0.001$ \\
\hline Respiratory rate (bpm) mean (SD) & $27.0(9.5)$ & $23.1(5.5)$ & $20.7(2.8)$ & $<0.001$ \\
\hline SPO2 median (IQR) & $95.0(17)$ & $99.0(3)$ & $100(2)$ & $<0.001$ \\
\hline BS median (IQR) & $8.8(6.8)$ & $7.8(3.1)$ & $7.9(3.4)$ & 0.102 \\
\hline LOS median (IQR) & 18.5(19.8) & $12.0(9)$ & $10.0(7)$ & 0.002 \\
\hline Mental status, n (\%) & & & & $<0.001$ \\
\hline Alert & 11 (15.9) & $166(79.8)$ & $103(97.2)$ & \\
\hline Reacting to voice & $7(10.1)$ & $29(13.9)$ & $3(2.8)$ & \\
\hline Reacting to pain & $20(29.0)$ & $12(5.8)$ & $0(0.0)$ & \\
\hline Unresponsive & 31 (44.9) & $1(0.5)$ & $0(0.0)$ & \\
\hline
\end{tabular}

Abbreviations: T Temperature, SBP Systolic blood pressure, DBP diastolic blood pressure, SPO2 Percutaneous oxygen saturation, BS blood sugar, LOS length of stay, IQR interquartile range, bpm, beats or breaths per minute.

Table 3 Logistic regression analysis of SZTS for in-hospital mortality, ICU admission and general ward admission

\begin{tabular}{|c|c|c|c|c|}
\hline \multirow[t]{2}{*}{ Triage Level } & \multicolumn{2}{|c|}{$\begin{array}{l}\text { Model 1, OR (95\% CI) P } \\
\text { value }\end{array}$} & \multicolumn{2}{|c|}{$\begin{array}{l}\text { Model 2, OR (95\% CI) P } \\
\text { value }\end{array}$} \\
\hline & Level 1 & Level 3 & Level 1 & Level 3 \\
\hline In-hospital mortality & $\begin{array}{l}11.6(5.8, \\
22.9)\end{array}$ & $0(0.0$, inf $)$ & $\begin{array}{c}11.8(5.8, \\
24.2)\end{array}$ & $0(0.0$, inf $)$ \\
\hline & $\square 0.001$ & 0.987 & $\square 0.001$ & 0.987 \\
\hline Admission to ICU & $\begin{array}{l}14.4 \\
(7.4,27.7)\end{array}$ & $\begin{array}{c}0.0 \\
(0.0,0.3)\end{array}$ & $\begin{array}{l}15.9(8.0 \\
31.4)\end{array}$ & $\begin{array}{r}0.0(0.0, \\
0.3)\end{array}$ \\
\hline $\begin{array}{l}\text { Admission to general ward } \\
\text { unit }\end{array}$ & $\begin{array}{l}\square 0.001 \\
10.6 \\
(1.4,79.2)\end{array}$ & $\begin{array}{c}0.002 \\
0.1 \\
(0.1,0.2)\end{array}$ & $\begin{array}{c}\square 0.001 \\
10.2(1.4 \\
76.4)\end{array}$ & $\begin{array}{r}0.001 \\
0.1(0.1 \\
0.2)\end{array}$ \\
\hline & 0.022 & $\square 0.001$ & 0.024 & $\square 0.001$ \\
\hline
\end{tabular}


Model 1, original model; model 2 with adjustment for age and gender. Presented as OR with 95\% CI (Level 2 as reference). ICU intensive care unit, SZTS Shenzhen Triage System.

Table 4 Association of triage level with in-hospital mortality, ICU admission and general ward admission

\begin{tabular}{cclll}
\hline Triage level & $\mathrm{n}$ & $\begin{array}{l}\text { Admission to general ward } \\
\mathrm{n}(\%)\end{array}$ & $\begin{array}{l}\text { Admission to ICU } \\
\mathrm{n}(\%)\end{array}$ & $\begin{array}{l}\text { In-hospital mortality } \\
\mathrm{n}(\%)\end{array}$ \\
\hline Level 1 & 69 & $68(98.6)$ & $53(76.8)$ & $35(50.7)$ \\
Level 2 & 208 & $180(86.5)$ & $39(18.7)$ & $17(18.1)$ \\
Level 3 & 106 & $46(43.4)$ & $1(0.9)$ & $0(0.0)$ \\
& & & & \\
\hline
\end{tabular}

\section{Figures}




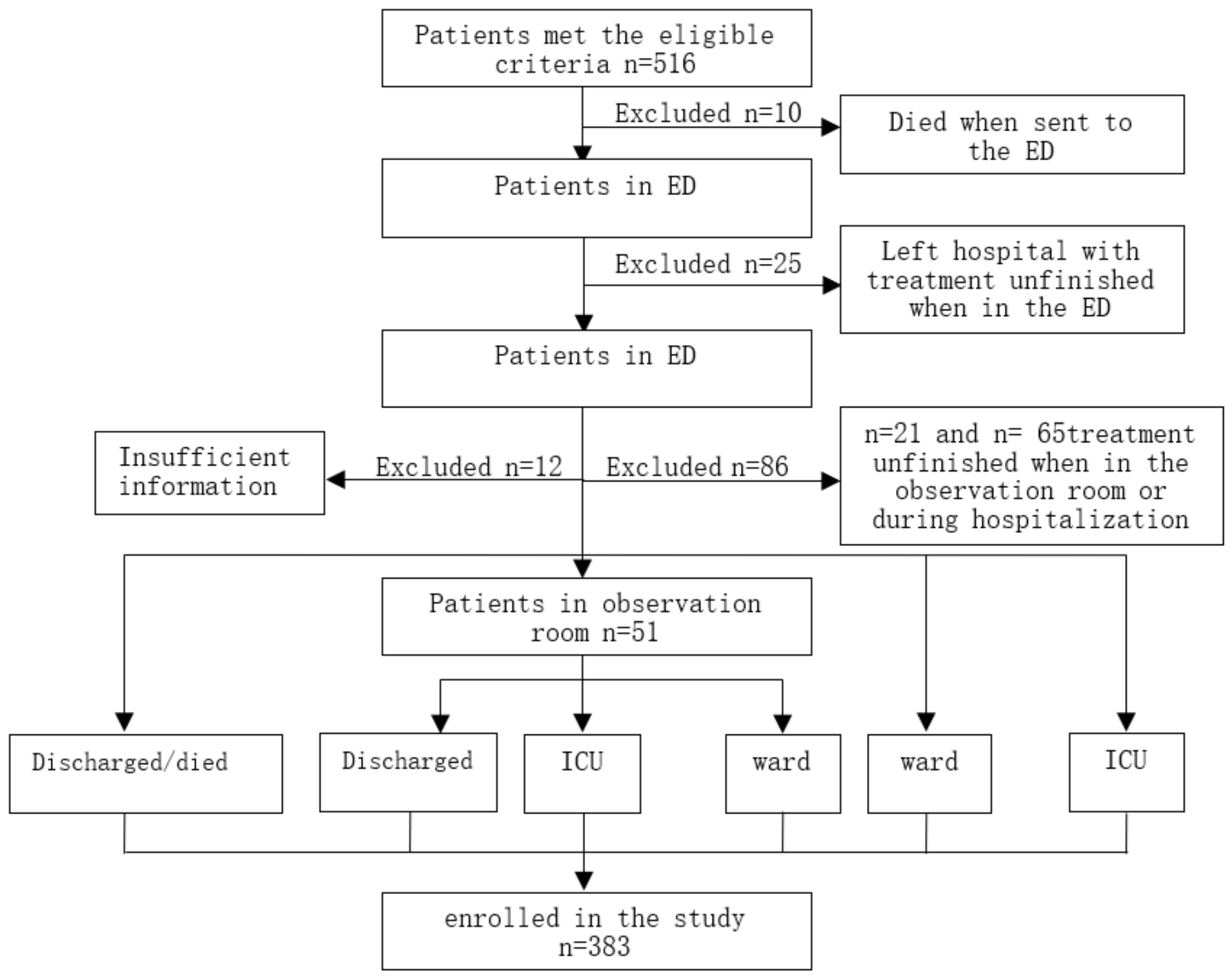

Figure 1

Patients who presented to the ED between May17, 2017 and September 27, 2017 were admitted. Abbreviations: ICU intensive care unit, ED emergency department. 


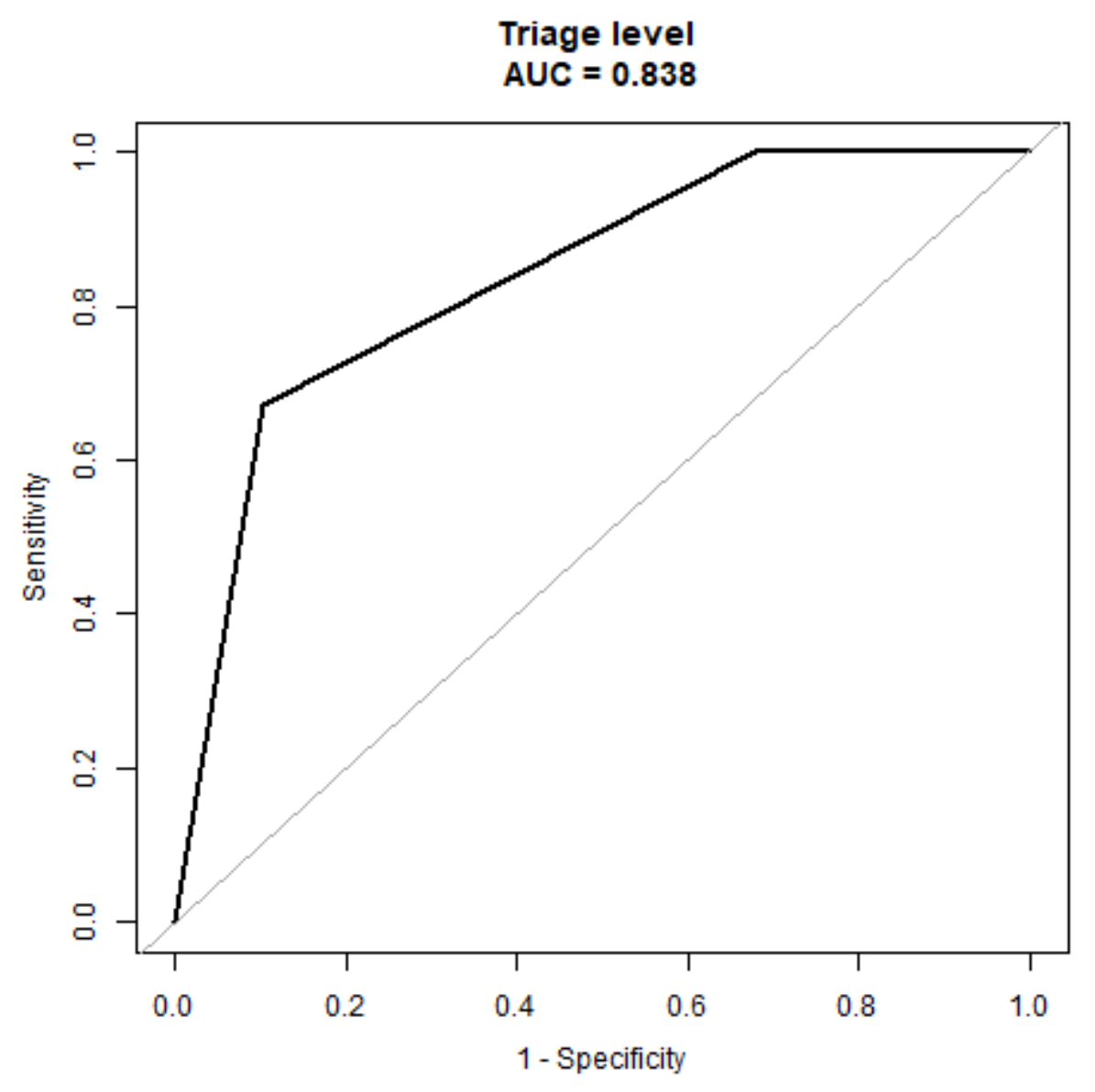

Figure 2

Figure 2, Figure 3, Figure 4. The area under the receiver operating characteristic curve (AUC) were measured to evaluate the predictive ability of the SZTS. Abbreviations: AUC - area under the receiver operating characteristic curve. 


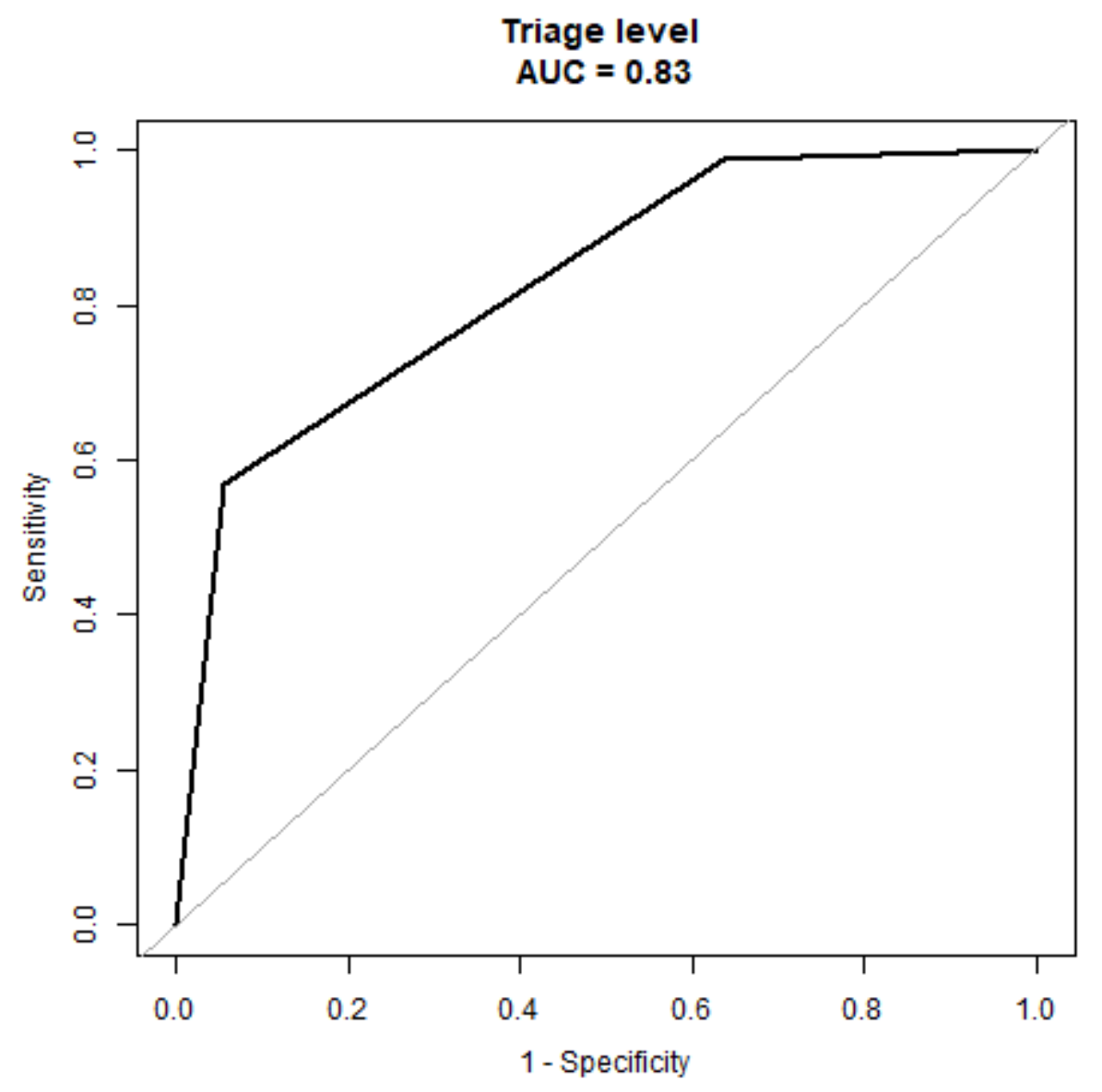

Figure 3

Figure 2, Figure 3, Figure 4. The area under the receiver operating characteristic curve (AUC) were measured to evaluate the predictive ability of the SZTS. Abbreviations: AUC - area under the receiver operating characteristic curve. 


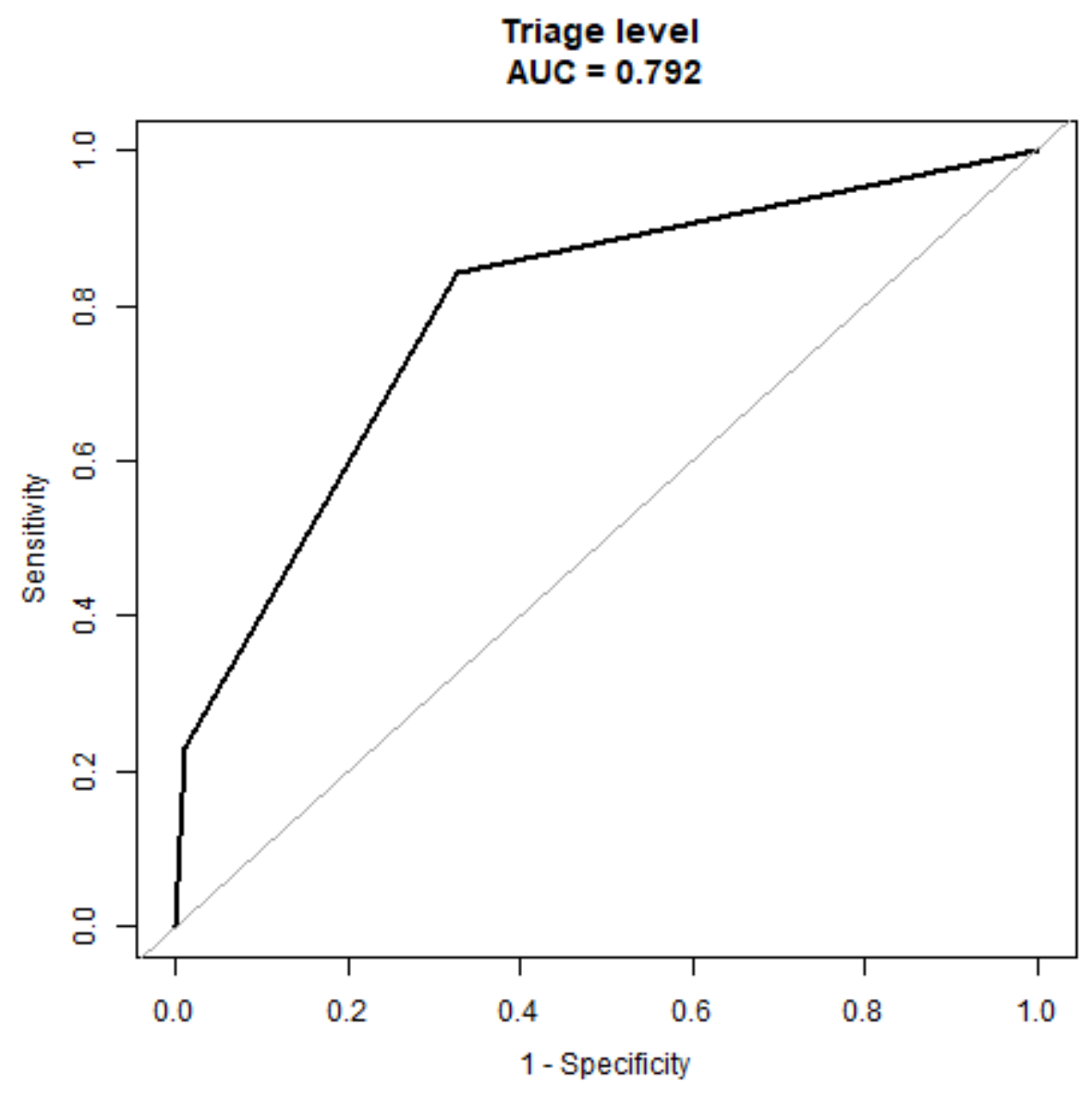

Figure 4

Figure 2, Figure 3, Figure 4. The area under the receiver operating characteristic curve (AUC) were measured to evaluate the predictive ability of the SZTS. Abbreviations: AUC - area under the receiver operating characteristic curve.

\section{Supplementary Files}

This is a list of supplementary files associated with this preprint. Click to download.

- STROBEchecklist.docx 